\title{
Verbal Reduplication and Grammaticalization: A Study of Mandarin VV-kan and V-kankan Constructions
}

\author{
YI-YANG CHENG
}

National Taiwan University, Taiwan

\section{Introduction}

The present study investigates two constructions in Mandarin ${ }^{1}-V V$-kan and $V$-kankan-which show an interesting formal asymmetry in the distribution of verbal reduplication and kan 'see', but denote the same meaning 'try ... and find out'. The VV-kan construction involves a schematic slot that is filled with a reduplicated verb, as in (1), while the V-kankan construction involves a verb that is followed by reduplicated kankan 'see.see', as in (2). What is intended to be discovered, or "found out," by trying out the action is either covertly implied through context, as in (1a) and (2a), or overtly expressed by an interrogative complement clause, as in (1b) and (2b). Despite the formal asymmetry between the two constructions, (1) and (2) are observed to be free variant forms competing for the same meaning 'try ... and find out', since a speaker would switch from one to the other without even noticing their choices.
(1a) zhe shui ni hehe-kan ba!
this water you drink.drink-see PART

'Try drinking this water and find out (implied: how it tastes)!'

\footnotetext{
${ }^{1}$ Contemporary data examined in this study are based on Mandarin spoken in Taiwan.
} 


\section{Yi-Yang Cheng}

(1b) zhe shui

$$
\begin{array}{lll}
\text { ni hehe-kan } & \text { weidao ruhe } \\
\text { you drink.drink-see } & \text { flavor how }
\end{array}
$$

this water

'Try drinking this water and find out how it tastes!'

$\begin{array}{rlll}\text { (2a) zhe shui ni he-kankan } & \text { ba! } \\ \text { this } & \text { water } & \text { you drink-see.see } & \text { PART }\end{array}$

'Try drinking this water and find out (implied: how it tastes)!'

$\begin{array}{rllll}\text { (2b) zhe } & \text { shui } & \text { ni he-kankan } & \text { weidao ruhe } \\ \text { this } & \text { water } & \text { you drink-see.see } & \text { flavor how }\end{array}$

'Try drinking this water and find out how it tastes!'

With evidence from corpus data, however, Cheng and Lu (2012) show that the two constructions can be distinguished based on the types of verbs that can fit into the schematic V/VV slot. ${ }^{2}$ While both constructions can be used with monosyllabic verbs, as has been shown in (1) and (2), V-kankan can also be used with disyllabic compound verbs, as in (3a), and other complex verb phrases, as in (3b) and (3c). That is, both morphologically simple and complex verbs occur in V-kankan. ${ }^{3}$

(3a) [jiancha] $]_{\text {comp-kankan }}$

(V-V compound)

examine-see.see

'try examining and find out'

(3b) [zuo shiyan $]_{\text {Vp-kankan }}$

(VO verb phrase)

[do experiment]-see.see 'try doing some experiment and find out'

(3c) [ba shiqing wancheng] $]_{\text {BA-kankan }}$

(ba construction $)^{4}$

[BA business finish]-see.see 'try finishing the business and find out'

Syntactically, transitive use of VV-kan can take both clausal and nominal complements, as can be seen in (1b) and (4a), but V-kankan only allows clausal complements, as in (4b), and a nominal complement would have to be

2 Cheng and Lu's (2012) finding is based on data retrieved from the Academia Sinica Balanced Corpus of Modern Chinese. All 141 tokens of $V V$-kan are used exclusively with monosyllabic verbs. There are 16 tokens of $V$-kankan used with morphologically complex verbs (14 compound verbs; 2 V-O phrases) and 7 used with monosyllabic verbs.

${ }^{3}$ Since the majority of morphemes in Chinese are monosyllabic (with only a few exceptions in loan words), multisyllabic words generally are morphologically complex (Norman 1988:156).

${ }^{4}$ The $b a$ construction in Mandarin involves the direct object being placed immediately after $b a$ and before the verb (Li and Thompson 1981:463). 


\section{Verbal Reduplication and Grammaticalization}

incorporated with the verb in $\mathrm{V}$ slot into a complex verb phrase that precedes kankan. Therefore, ta 'he' serving as wen-kankan's complement in (4b) is unacceptable, but the complex verb phrase wen ta 'ask him' in [wen ta]-kankan 'try asking him and find out' in (4c) is acceptable.

$\begin{array}{clll}\text { (4a) wo } & \text { qu } & \text { wenwen-kan } & \text { ta } \\ \text { I } & \text { go } & \text { ask.ask-see } & \text { he }\end{array}$

'I'll go and try asking him and find out.'

(4b) wo qu wen-kankan ?[ta $]_{\mathrm{NP}} /[\text { ta yuan-bu-yuanyi lai }]_{\mathrm{CL}}$

I go ask-see.see he/he willing-NEG-willing come

'I'll go and try asking him/and find out if he's willing to come.'

(4c) wo qu [wen [ta $\left.]_{\mathrm{NP}}\right]-k a n k a n[(\mathrm{ta}) \text { yuan-bu-yanyi lai }]_{\mathrm{CL}}$

I go ask he-see.see (he) willing-NEG-willing come

'I'll go and try asking him and find out if he's willing to come.'

In addition, V-kankan exhibits ambiguity in two contexts. When preceded by a complex motion verb, kankan exhibits a fuzzy status between (i) an independent verb in serial verb construction [ $\left.\mathrm{V}_{\text {compx/motion }}+k a n k a n\right]_{\mathrm{svc}}$ meaning 'to go somewhere to look around/take a look,' as in (5a) $)^{5}$, and (ii) a grammaticalized

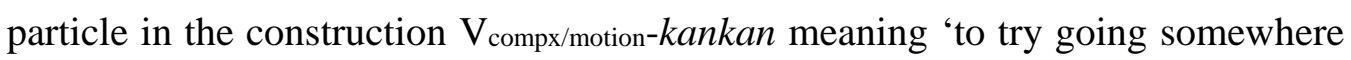
and find out,' as in (5b).

(5) wo xiangyao chuguo(-)kankan
I want go.abroad(-)see.see
a. 'I want to go abroad to look around/take a look (of the world).'
b. 'I want to try going abroad and find out.'

When kankan is preceded by a complex verb and followed by an interrogative complement clause, the collocation $\left[\mathrm{V}_{\text {compx }}+\right.$ kankan $\left.+\mathrm{CL}_{\text {intrgv }}\right]$ has two interpretations: (i) 'to do something to find out what happens', with $\mathrm{V}_{\text {compx }}$ and kankan + CLintrgv serving as two verbs in serial verb construction, as in (6a); (ii)

5 Mandarin serial verb constructions denoting two or more separate events are essentially ambiguous (Li and Thompson 1981:595). Here, a purpose relation between two events is the most appropriate interpretation, hence the translation ' $\mathrm{V}_{1}$ to $\mathrm{V}_{2}$ '. 


\section{Yi-Yang Cheng}

'to try doing something and find out what happens' with $\mathrm{V}_{\text {compx }}$-kankan serving as a complement-taking construction meaning 'try ... and find out', as in (6b).

(6) zhe ben shu ni naqu cankao(-)kankan you-mei-you bangzhu This CL book you take.go reference(-)see.see exist-NEG-exist help

a. 'Take this book as reference to find out if it can be of any help.'

b. 'Try taking this book as reference and find out if it can be of any help.'

The two constructions raise particular interests because they show differences in their morpho-syntactic properties but are functionally equivalent when used with monosyllabic verbs. $V$-kankan can be used with both simplex and complex verbs, is syntactically constrained, and shows ambiguity in two contexts when preceded by complex verbs, while $V V$-kan shows a more stable behavior-it is used exclusively with simplex verbs, and takes both nominal and clausal complements.

In light of their synchronic properties as discussed above, this study is an attempt to examine the grammaticalization pathways that the two constructions have undergone by exploring uses of kan, kankan, and their diachronic evolution in Middle, Pre-Modern, and Modern Chinese. ${ }^{6}$ It is demonstrated that although grammaticalization of the two constructions occurred in different morpho-syntactic environments at different historical stages in Chinese, a recurrent path has been taken first by $V V$-kan, and later by $V$-kankan which is right now emerging in Contemporary Mandarin. Taking into account all the interacting factors that have led to a synchronic competition of the two constructions, the phenomenon investigated then presents language as a complex adaptive system (The "Five Graces Group” 2009), in which change is motivated by interacting language-internal and language-external factors (Du Bois 1985).

This paper is organized as follows. A diachronic account of the two constructions is given respectively in section 1 and section 2 . In section 3 , their synchronic coexistence is examined with regard to interacting language-internal and external factors, and in section 4 , the conclusion of this study is provided.

\footnotetext{
6 The diachronic data examined in this study are retrieved from the Scripta Sinica database unless otherwise indicated. The periodicization of retrieved data generally follows Chen (1999:2) with minor modifications: (i) the period Medieval Chinese is referred to as Middle Chinese; (ii) a new period Contemporary Chinese is added to capture further development of the two constructions from their sources in Pre-Modern and Modern Chinese.
} 


\section{Verbal Reduplication and Grammaticalization}

\section{Verbal Reduplication and the Development of VV-kan}

As has been discussed in literature, the $V V$-kan construction is diachronically related to the V-yi-V construction which consists of a head verb $\mathrm{V}$, and $y i-\mathrm{V}$ which functions like an adverbial phrase denoting the meaning 'once' (Chao 1968: 205). The construction as a whole has the literal meaning 'to do something just once' but may also be metaphorically interpreted as 'to do something a little:'

(7) shuo-yi-shuo (say-one-say) 'say once say a little'

Although still in debate, $\mathrm{V}-y \mathrm{i}-\mathrm{V}$ is generally considered as one of the diachronic sources that led to contemporary verbal reduplication (Wu 1995:165). First attested in early Pre-Modern Chinese, V-yi-V had undergone the drop of yi 'one' in the path $\mathrm{V}-y i-\mathrm{V}>\mathrm{VV}$, and grammaticalized into $\mathrm{VV}$, through which the metaphorical sense 'to do just a little' was conventionalized and came to be known as the tentative aspect in contemporary verbal reduplication ${ }^{7}$ (Smith 1994:119). As can be seen in (8), reduplicated verbs cast a tentative scope over the designated event, hence $d a$ 'play' becoming dada 'play some/just a little'.

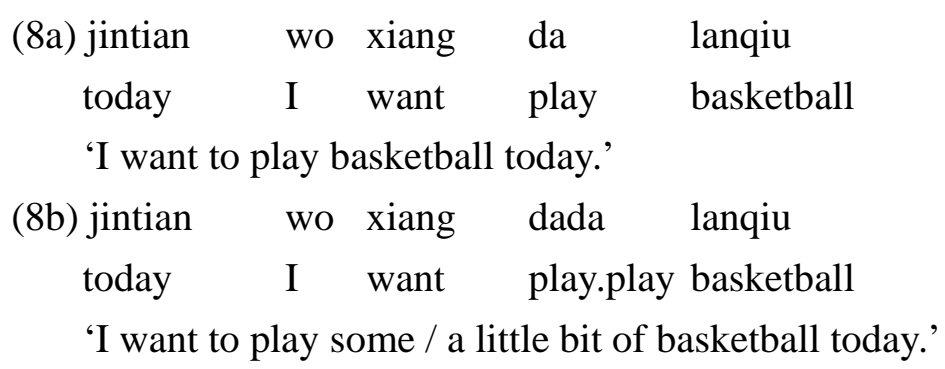

What came to be associated with the V-yi-V construction was post-verbal kan during Pre-Modern Chinese. During Middle Chinese, the meaning of kan ranged from perception 'look/see' to cognition 'find out/check'. The former is restricted to situations where the object/event being 'looked at/seen' is concrete or has

\footnotetext{
${ }^{7}$ Note that an optional yi 'one' may still be inserted into reduplicated verbs in contemporary Chinese. Therefore, the path $\mathrm{V}-y i-\mathrm{V}>\mathrm{VV}$ does not mean that all instances of $\mathrm{V}-y i-\mathrm{V}$ grammaticalized into VV in Mandarin. It only refers to one of the diachronic sources leading to contemporary verbal reduplication (cf. the origin of kankan discussed in the next section).
} 


\section{Yi-Yang Cheng}

already happened, while the latter is related to future-reference, or unknown nature of the object/event. Regarding their distribution (as main predicate or post-verbally in serial verb construction) and syntactic behaviors (transitivity), Middle Chinese kan can be categorized into the following four types:

(9a) Main predicate/transitive/perceptual

$\left(k n_{v i .}+\mathrm{NP}\right)$

... kan xin fu

(ShiShuoXinYu)

... see new woman

'... see the bride.'

(9b) Main predicate/transitive/cognitive

$$
\left(k a n_{v t .}+\text { CLintrgv }\right)
$$

... kan tang re bu

(Wu 1995:162)

... see souphot NEG

'... find out if the soup is hot or not.'

(9c) SVC/intransitive/perceptual

$\left(\mathrm{V}_{\text {motion }}+\mathrm{kan}_{\text {vi. }}\right)$

xin fei shu chu kan

(ShiShuoXinYu)

Xin abandon book out see

'Xin then stopped studying and go out to see (what has happened).'

(9d) SVC/intransitive/cognitive

$\left(\mathrm{V}_{\text {action }}+\right.$ kan $\left._{\text {vi. }}\right)$

ni shi shuo kan
you try say see

(ZuTangJi)

'Try saying (it) and find out (what will happen).'

Note that the case in (9d) is usually found in imperatives, where a pragmatic implicature 'try' naturally arises: as the speaker requests that the hearer perform some action in order to find out what will happen as a result of that action, s/he is implicitly requesting the hearer to try performing that action. Therefore, 'try' is implied in (9e) below, even when the lexical verb shi 'try' is not present:

\footnotetext{
(9e) qing xong yu wu nian kan!

(ZuTangJi)

please brother to I read see

'Please, my brother, read it / (try) reading it to me to find out!'
}

It was post-verbal intransitive kan with the cognitive sense 'find out' in (9d) and (9e) that came to co-occur with V-yi-V during Pre-Modern Chinese. As shown in 


\section{Verbal Reduplication and Grammaticalization}

(10), the collocation [V-yi-V + kan] involves 'try' as its meaning even when the verb shi 'try' is absent and when occurring in declaratives:
(10) die
qi zhitou suan-yi-suan kan
(ErKePaiAnJingQi)
pile up finger count-one-count see

'(He) piled up his fingers to try counting in order to find out.'

Tsao (2001:291) observes a close semantic connection between 'do a little' and 'try': "when one is just trying, one will normally do just a little." Accordingly, 'try' implied by post-verbal kan may have been pragmatically strengthened by the semantics of $\mathrm{V}-y i-\mathrm{V}$, hence conventionalized in the context $[\mathrm{V}-y i-\mathrm{V}+k a n]$. As $\mathrm{V}-y i-\mathrm{V}$ grammaticalized into $\mathrm{VV}$, the collocation [VV + kan] was also found in Modern Chinese:
(11) gu
chacha kan
tentatively check.check see
(LuQiDeng)
'(I’ll) just try checking and find out.'

Note that by Modern Chinese, all cases of post-verbal kan were used intransitively. The transitive uses of $V V$-kan, as seen in (1) and (4a), are clearly a contemporary innovation, which indicates that (i) the meaning 'try $\cdots$ and find out' has been semanticized in [VV + kan]; (ii) a constructional schema $V V$-kan with the new coded meaning has emerged; and (iii) the grammaticalized construction has generalized its grammatical behavior to be used transitively in taking nominal and clausal complement, ${ }^{8}$ hence is not restricted to the original context.

(12) Development of $V V$-kan from post-verbal kan

$$
\begin{array}{lll}
\text { Mid. Ch. } & \text { PreMdn.-Mdn. Ch. } & \text { Contemp. Ch. } \\
\mathrm{V}_{\text {motion }}+k a n_{v i .} & & \\
\mathrm{V}_{\text {action }}+k a n_{v i .} & >\quad \mathrm{V}-\mathrm{yi}-\mathrm{V}+\quad k a n_{v i .}>\mathrm{VV}+k a n_{v i .}>\quad V V-k a n_{v i . v v t}
\end{array}
$$

$$
\text { ‘try' as pragmatic implicature }>\text { 'try' as conventionalized implicature } \quad>\text { 'try' as new coded meaning }
$$

\footnotetext{
${ }^{8}$ Note that kan taking interrogative clauses as seen above in (9b) is still in use. The transitive use of $V V$-kan that takes an interrogative clause as its complement might be attributed to analogy to complement taking verbal kan.
} 


\section{Yi-Yang Cheng}

\section{Reduplicated kankan, Complex Verbs, and the Emerging V-kankan}

V-kankan interestingly contrasts with $V V$-kan since the former involves post-verbal reduplicated kankan instead of a schematic slot filled with reduplicated verbs. However, unlike VV in VV-kan, kankan was among the verbs that underwent direct reduplication instead of the path $\mathrm{V}-y i-\mathrm{V}>\mathrm{VV}$, as indicated by Tsuei (2010). In Middle Chinese, direct reduplication generally rendered a durative/continuous interpretation, and reduplicated kankan served like an adjunct adverbial clause with the meaning 'as someone looks/observes for a while', as in (13a). However, when followed by a nominal complement, reduplicated kankan came to have the meaning 'to look at', and when used in imperatives it may also servea tentative meaning 'to take a look', as can be seen in (13b).

(13a) Intransitive kankan 'durative see'

$\begin{array}{llll}\text { kankanshui } & \text { mo } & \text { lai } & \text { shi lu (Tsuei 2010:228) } \\ \text { see.see water } & \text { submerge } & \text { come } & \text { time road }\end{array}$

'As (I) looked for a while, the water has submerged the road I took.'

(13b) Transitive kankan + NP 'durative look at/tentative take a look at'

$\begin{array}{ll}\text { kankanjia shang } & \text { ying } \\ \text { see.see rack up } & \text { eagle }\end{array}$

(Tsuei 2010:228)

'Look at/take look at the eagle on the rack!'

In Pre-Modern Chinese, the tentative meaning of transitive kankan was extended to several different contexts. Like kan in Middle Chinese, kankan at this period involved both perceptual and cognition senses. First, it began to take interrogative clausal complements and had the cognition meaning 'find out'. Second, it began to occur in post-verbal positions in a serial verb construction, where the first verb can be classified into two categories: motion verbs and action verbs, as can be seen in (14). ${ }^{9}$ Note that in (14c) there is a pragmatic implicature 'try' arising out of the context, since when one is doing something in order to find out what will happen as a result of performing that action, one is also trying that action out.

\footnotetext{
9 There are also cases of kankan used as main predicate, but their functions are identical to those of their post-verbal counterparts, thus are not included due to spatial concerns.
} 


\section{Verbal Reduplication and Grammaticalization}

(14a) SVC/transitive/perceptual ( $\mathrm{V}_{\text {compx }}+$ kankan $\left.+\mathrm{NP}\right)$

xiaozi jinwan yao huiqu kankan jiali (XingShiHengYan)
little.boy tonight will return.go see.see home.inside
'The little boy is going to go back to take a look at/check on his family.'

(14b) SVC/intransitive/perceptual ( $\mathrm{V}_{\text {motion/compx }}+$ kankan)

$\begin{array}{llll}\mathrm{xu} & \text { shangxian } & \text { qinqu } & \text { kankan (XiYouJi) } \\ \text { need } & \text { up.celestial } & \text { personally.go } & \text { see.see }\end{array}$

'It requires that the great celestial go and take a look in person.'

(14c) SVC/transitive/cognition ( $V_{\text {compx }}+$ kankan $\left.+\mathrm{CL}_{\text {intrgv }}\right)$

$\begin{array}{llllll}\text { ni taitou kankan wo shi nage } & \text { (XiYouJi) } \\ \text { you lift.head see.see } & \text { I be which.one } & \end{array}$

'(Try) lifting your head/lift your head to find out which one I am!'

Also note that the verbs preceding Modern Chinese kankan were usually morphologically complex. This can be attributed to a long period of development of complex predicates that had been in progress starting from the period of Middle Chinese. ${ }^{10}$ The newly formed complex verbs constitute a crucial factor that distinguishes the development of $V$-kankan from that of $V V$-kan, which will be discussed in the next section. Right now, it is appropriate to see the connection between the collocation [ $\mathrm{V}_{\text {compx }}+$ kankan $\left.+\mathrm{CL}_{\text {intrgv }}\right]$ in Pre-Modern Chinese and its counterpart in Contemporary Chinese. ${ }^{11}$.

The reader is advised to refer back to (6) and compare it with (14c). Observe that while 'try' had been an implicature in (14c), it is included in one of the two meanings in the ambiguious $\left[\mathrm{V}_{\text {compx }}+\right.$ kankan $\left.+\mathrm{CL}_{\text {intrgv }}\right]$ in (6), where one interpretation is identical to the coded meaning in (14c), and the other interpretation includes 'try' serving no longer as an implicature but as part of the new coded meaning. According to Hopper (1991), grammaticalization crucially involves inferences being semanticized in a forming construction, the process of which may last for a long period where the old sense and the semanticizing inference coexist. Since there is evidence that $\left[\mathrm{V}_{\text {compx }}+k a n k a n+\mathrm{CL}_{\text {intrgv }}\right]$ is

\footnotetext{
${ }^{10}$ Multisyllabic complex verbs began to develop as the number of possible syllables had been decreasing due to simplification of the Chinese phonological system, before which the language had shown a one-word/one-syllable pattern (Norman 1988:112).

11 No obvious changes are found in Modern Chinese $\left[\mathrm{V}_{\text {compx }}+\right.$ kankan $\left.+\mathrm{CL}_{\text {intrgv }}\right]$.
} 


\section{Yi-Yang Cheng}

attested in both Pre-Modern and Contemporary Chinese, it can be inferred that the implicature 'try' is right now undergoing semanticization in this context.

(15) Semanticization of 'try' in [V $\mathrm{V}_{\text {compx }}+$ kankan + CLintrgv $]$

PreMdn.-Mdn. Ch.

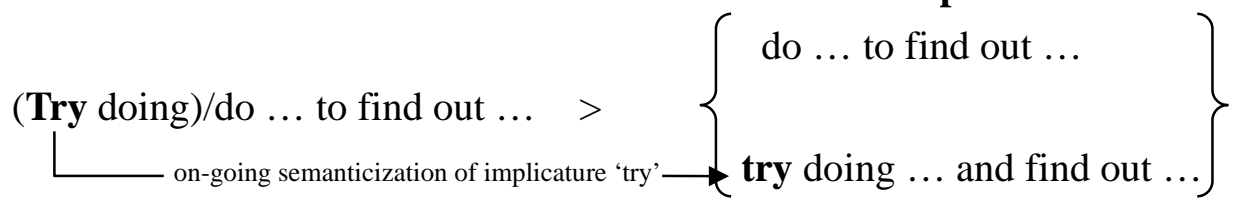

Having established that there is an on-going process of semanticization in [ $\mathrm{V}_{\text {compx }}$ + kankan $\left.+\mathrm{CL}_{\text {intrgv}}\right]$, the other cases of kankan-related constructions (which are treated as instantiations of $V$-kankan in the introduction) exemplify a typical case of layering (Hopper 1991), where older and newer forms co-exist at an intermediate stage of grammaticalization. First, the fact that there is intransitive use of $\mathrm{V}_{\text {compx }}-k a n k a n$, as seen in (3) indicates that a gradual reanalysis [ $\left.\mathrm{V}_{\text {compx }}\right]+$ $\left[\right.$ kankan $\left.+\mathrm{CL}_{\text {intrgv }}\right]>\mathrm{V}_{\text {comp-kankan }}$ (+ $\mathrm{CL}_{\text {intrgv }}$ ) is in progress. Second, the other

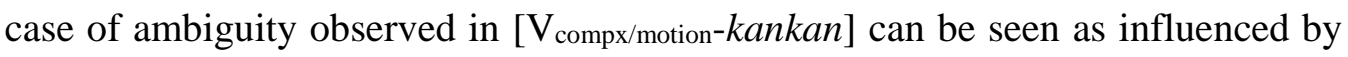
the forming construction V-kankan, whose meaning 'try $\cdots$ and find out' is being analogically mapped onto the collocation $\left[\mathrm{V}_{\text {compx/motion }}+\right.$ kankan] where 'try' did not exist during Pre-Modern Chinese. Third, as V-kankan is forming, it is also spreading its use to monosyllabic verbs, as a result of generalization of its grammatical behavior. ${ }^{12}$ Hence a competition with the extant $V V$-kan is set up.

(16) Layering and the emerging V-kankan

PreMdn.-Mdn. Ch.

Contemp. Ch.

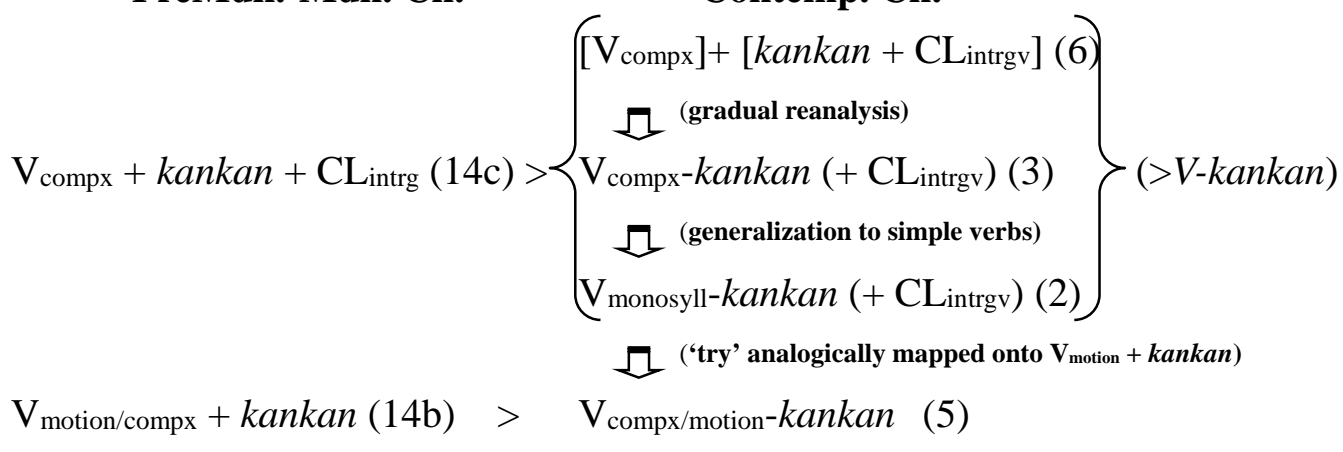

12 Note that no uses of kankan preceded by monosyllabic verbs with the meaning 'try ... and find out' were attested before Contemporary Chinese. 


\section{Verbal Reduplication and Grammaticalization}

The on-going change is explanatory of the syntactic constraint that V-kankan does not take nominal complements, as has been shown in (4b). This can be seen as a case of persistence (Hopper 1991) of original context since the collocation [ $\mathrm{V}_{\text {compx }}$ + kankan + NP], as in (14a), did not involve implicature 'try' at all. Therefore, $V$-kankan is different from $V V$-kan in that the former is an emerging construction that is still undergoing grammaticalization, while the latter has developed into a full-fledged construction with its own generalized grammatical behavior.

\section{A Recurrent Path and Co-existence of the Two Constructions}

As has been shown, different morpho-syntactic contexts are observed in the grammaticalization of VV-kan and V-kankan: while the former involved intransitive kan that participated in the path $\mathrm{V}-y i-\mathrm{V}>\mathrm{VV}$, the latter is emerging from the context $\left[\mathrm{V}_{\text {compx }}+\right.$ kankan $\left.+\mathrm{CL}_{\text {intrgv }}\right]$ svc. A close examination of the development indicates that their convergence in Contemporary Chinese is nevertheless motivated and can be attributed to a recurrent path that has been taken.

To begin with, the interaction of two historical factors-development of verbal reduplication and formation of complex predicates-plays a crucial role in providing context for the emergence of $V$-kankan. As mentioned above, the lexical source for $V$-kankan, i.e. reduplicated kankan, is created as distinct from those for $V V$-kan since the development of contemporary verbal reduplication can be seen as a merger of two diachronic paths into one synchronic morphological process.

(17) Two merging paths of verbal reduplication

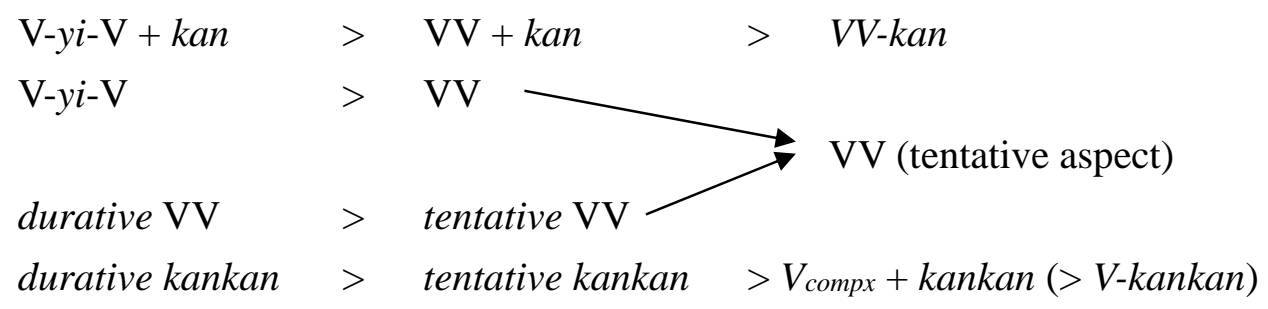

On the other hand, the context for V-kankan's emergence crucially relies on the development of complex predicates. By the period of Modern Chinese, post-verbal monosyllabic kan had gradually lost its function as an independent 


\section{Yi-Yang Cheng}

verb since it has become highly dependent on the preceding VV in denoting the sense 'try ... and find out' in the evolution of $V V$-kan. At the same period, kankan, whose tentative use had been well-developed, had also started to interact with newly formed complex verbs and developed periphrastic combinations that served to convey concepts that used to be expressed by post-verbal kan in Middle Chinese. As illustrated in (18), two uses of post-verbal kan in Middle Chinese have corresponding (near) synonymous uses of post-verbal kankan in Modern Chinese. Observe that the two stages are separated by a long period of development of complex predicates in the language.

(18) Middle Chinese

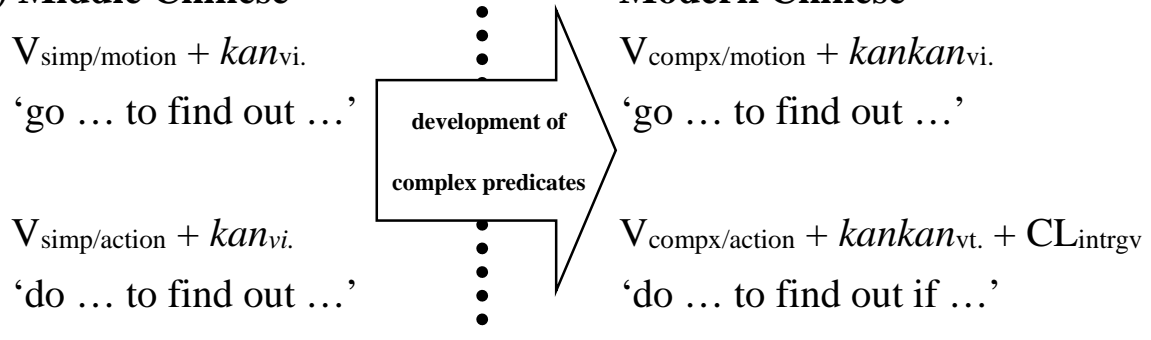

Furthermore, comparison of the development of the two constructions revealsthat the mechanisms involved are essentially the same despite the different, unpredictable morpho-syntactic contexts involved. Considering the nature of the lexical sources, kan and kankan at all levels both had the cognitive sense 'find out' from the perceptual sense 'see/look,' as a result of cross-linguistically attested metaphorical mapping between the domains of perception and cognition (Sweetser 1990). One characteristic of the rise of the meaning 'find out' from 'see/look' is future time-reference; that is, kan/kankan meaning 'find out' both refer to events that are unactualized but of interest to the interlocutors because they are to be actualized as a result of performing certain actions. Interacting with context, the meaning 'find out' then gave rise to the pragmatic implicature 'try' through metonymic inferencing (Hopper and Traugott 2003). As the implicature 'try' became conventionalized through pragmatic strengthening, and the context for the rise of the implicature have grammaticalized, the construction as a whole with 'try' semanticized as part of its meaning ceased to be restricted by the original context and developed its own grammatical behavior. The previous two sections have been devoted to accounting for the observation that synchronically 


\section{Verbal Reduplication and Grammaticalization}

$V V$-kan has reached this final stage in which 'try' has been semanticized as new coded meaning, while $V$-kankan is right now entering this stage.

Considering the preceding discussions, the interaction of diachrony with cognitive mechanisms therefore leads to a recurrent path that has been taken first by post-verbal kan in the grammaticalization of $V V$-kan, and later by post-verbal kankan in the emerging V-kankan.

(19) Mechanisms involved in the recurrent path

$$
\text { 'see' } \rightarrow \text { 'find out' }>\quad \text { '(try) ... to find out' } \quad>\quad \text { 'try ... and find out' }
$$

Metaphor

Perception $\rightarrow$ Cognition

\section{Metonymic Inferencing Pragmatic Strengthening} invited inference of 'try' conventionalization of 'try'

The recurrent path can therefore be seen as a strange attractor that yields a repeated global pattern despite showing differences in detail (Bybee 2010:198). In light of this, coexistence of the two constructions at the synchronic level is motivated by the interaction between language-internal factors (the development of verbal reduplication and complex predicates) and language-external factors (cognitive mechanisms of metaphor and metonymic inferencing). Note that the factors contributing to the coexistence of the two constructions are not independent but serve as different facets of the whole process of change, and that this process naturally leads to a competition of $V V$-kan and V-kankan at the synchronic level. ${ }^{13}$ The phenomenon then highlights the adaptive nature of language as a self-organizing complex system, in that it undergoes change as a result of the interaction of its internal organization with external environments (Du Bois 1985:362, The “Five Graces Group” 2009:16).

\section{Conclusion}

The present paper has explored the diachronic development of two constructions in Mandarin- $V V$-kan and V-kankan. It has been shown that the formal asymmetry between the schematic V/VV slot and kan/kankan underlying the two constructions is attributed to the different morpho-syntactic contexts involved in

13 Compare the competition between two coexisting negative constructions in English and their diachronic development (Bybee 2010:110). 


\section{Yi-Yang Cheng}

grammaticalization, and that a recurrent path has led to synchronic coexistence of the older $V V$-kan with the younger, still emerging V-kankan. The phenomenon examined presents language as a complex adaptive system, in which global patterns emerge from the complex interaction of internal and external factors.

\section{References}

Bybee, Joan. 2010. Language, Use, and Cognition. New York: Cambridge University Press.

Chao, Yuan Ren. 1968. A Grammar of Spoken Chinese. Berkeley: University of California Press.

Chen, Ping. 1999. Modern Chinese: History and Sociolinguistics. Cambridge: Cambridge University Press.

Cheng, Yi-Yang and Chiarung Lu 2012. Verbal Reduplication and Grammaticalization: A Corpus-based Study on Mandarin VV-kan and V-kankan Constructions. Proceedings of the $12^{\text {th }}$ Annual Meeting of the Japanese Cognitive Linguistics Association.

Du Bois, John. W. 1983. Competing Motivations. In J. Haiman, ed., Iconicity in Syntax. Amsterdam: Benjamins.

Hopper, Paul J. 1991. On Some Principles of Grammaticalization. In E. Traugott and B. Heine eds., Approaches to grammaticalization. Amsterdam: Benjamins.

Hopper, Paul. J. \& Elizabeth C. Traugott. 2003. Grammaticalization. Cambridge: Cambridge University Press.

Norman, J. 1988. Chinese. Cambridge: Cambridge University Press.

Li, Charles N. \& Sandra A. Thompson. 1981. Chinese Grammar: A Functional Reference. Berkeley: University of California Press. 


\section{Verbal Reduplication and Grammaticalization}

Smith, Carlota S. 1994. Aspectual Viewpoint and Situation Type in Mandarin Chinese. Journal of East Asian Linguistics 3:107-146.

Sweetser, Eve. 1990. From Etymology to Pragmatics: Metaphorical and Cultural Aspects of Semantic Structure. UK: Cambridge University Press.

The "Five Graces Group,” Clay Beckner, et al. 2009. Language Is a Complex Adaptive System: Position Paper. Language Learning 59(1):1-26.

Tsao, Feng-fu. 2001. Reduplication in Mandarin and Taiwan Southern Min. In H. Chappell, eds., Chinese grammar: synchronic and diachronic perspectives, 285-308, New York: Oxford University Press.

Tsuei, Yingxian. 2010. Tang Sung Yuan shiqi de “kankan” [kankan in the Tang, Sung, and Yuan periods]. Henan shifan daxue xuebao [Journal of Henan Normal University] 37(5):226-29.

Wu, Fuxiang 1995. Changshi tai zhuci “kan” de Lishi kaocha [Historical research on the tentative particle "kan"]. Yuyan Yanjiu [Linguistic research] 2: 161-166.

Yi-Yang Cheng

National Taiwan University, Taipei

Graduate Institute of Linguistics

1 Roosevelt Road

Taipei, Taiwan 10617

chengyiyang@outlook.com 